EDITORIAL

\section{Rigorous software engineering}

\author{
Sabine Glesner
}

Published online: 21 September 2013

(C) Springer-Verlag Berlin Heidelberg 2013

It is my pleasure to present this special issue on "Rigorous Software Engineering" in which we see the contributions from five well-known researchers who describe the work that they are doing in their research grants of the European Research Council (ERC). ERC research grants, which are given away by the European Commission, have gained a very well-known reputation. In this special issue, we have papers about ERC Starting Grants, which support young researchers, as well as papers about ERC Advanced Grants, which are open for excellent established researchers. All of the five researchers work in the field of software engineering and are concerned with methods to construct and maintain software systems that evolve over time, are safety-critical, work in embedded contexts, that we all know very well from our daily lives.

The origins of this special issue date back to a workshop that was held in Venice in September 2011, at which the five ERC grant holders got together to discuss their research agenda and their initial achievements. As one of the results of this workshop, the five principal investigators decided to work together on a special issue for "Computer ScienceResearch and Development" (CSRD) in which they present their ERC projects. Their papers provide an overview of the goals and motivations of the respective projects, and possibly also, for the more mature projects, of their achievements, in a way that might be of interest for a general audience, rather than a more specialist treatment of the subject matter. Special thanks go to Gerti Kappel from the Technical University of Vienna, who strongly encouraged and invited the ERC researchers to work on this special issue of CSRD.

S. Glesner $(\varangle)$

Technical University of Berlin, Berlin, Germany

e-mail: sabine.glesner@tu-berlin.de
As you can see from the papers in this special issue, there are still many severe problems when it comes to software engineering, although the origins of software engineering and the wish to do so correctly and efficiently date back already several decades. Nowadays, we see software that ranges from rather small applications in the embedded domain up to large systems that span world-wide over the internet. In this special issue, representatives of these various kinds of software are presented and their specific problems as well as the novel solution approaches of the ERC grant holders are described.

In detail, this issue presents the following five papers (in the same order as the authors gave their presentation at the initial workshop in 2011):

The first paper is by Carlo Ghezzi and his co-author Luciano Baresi from the Politecnico di Milano, Italy, and deals with self-managing software systems within the context of the ERC Advanced Grant on "Self Managing Situated Computing". Starting from the observation that modern software systems need to work in continuously changing environments, the authors develop a framework in which software systems can take an active role in the sense that they detect changes and react to them autonomously. Moreover, to make sure that the systems still behave correctly, the authors also include a life-long verification into their framework. In doing so, software systems shall be able to act and react in cyber-physical systems, where the physical and the cyberworld are increasingly growing together.

The second paper is by Sebastian Uchitel and his coauthors from the Imperial College in London. It discusses how model-based software engineering can be done without the need to have complete models already right in the beginning. Rather, within the ERC Starting Grant "Partial Behaviour Modelling", this approach provides for an iterative development of incomplete descriptions. For this pur- 
pose, partial behavior models are investigated. Furthermore, controller synthesis techniques are developed such that models of system requirements can be automatically built which guarantee system goals under specific environment assumptions. Moreover, automated diagnosis and refinement are studied with the goal to be able to handle partial or inconsistent models. And, finally, novel abstraction techniques are combined with human interaction to detect issues in behavior specifications and code.

In the third paper, which is by Marta Kwiatkowska from the University of Oxford, Great Britain, the vision of Everyware Verification is presented. She investigates everyware kind of systems, which are a sequel to software systems in the setting of ubiquitous computing. Computers disappear as they are integrated in everyday objects like clothing, equipment and even our bodies. This imposes severe safety requirements and, in turn, the need for suitable verification methods. In her ERC Advanced Grant "Veriware", Marta Kwiatkowska wants to answer principal questions around everyware verification, namely what models are appropriate, what kind of interaction, cooperation, and compositionality mechanisms are suitable, what correctness criteria apply, how non-functional properties such as e.g. resource usage can be measured, and if everyware verification is feasible, both in theory and practice. To answer these questions, Marta Kwiatkowska works on discrete and continuous-time probabilistic models, develops model checkers for cooperative behavior as well as quantitative verification methods for ubiquitous (e.g. implantable or wearable) devices, and shows how compositional quantitative verification as well as quantitative runtime verification can succeed.

In the fourth paper, John Mylopoulos from the University of Trento, Italy, and his co-authors present an approach for requirements-driven software evolution. Within the ERC Advanced Grant "Lucretius: Foundations for Software Evolution", starting from the observation that requirements on software systems very often evolve over time, a methodology is developed to deal with requirement changes systematically. For this purpose, they introduce evolution requirements that specify changes to other requirements when certain conditions apply. Furthermore, they show how such requirements can be modeled and operationalized at run time so that systems can be adapted dynamically. In particular, they identify a set of generic evolution operations and their effect on a given target system and the adaptation framework, whereas the target system is supposed to implement an interface for the evolution operations. In a case study involving an adaptive computer-aided ambulance dispatch, it is shown that the approach of requirements-driven software evolution adapts software systems well to new requirements.

Finally, the fifth paper by Tom Henzinger from the Institute of Science and Technology, Austria, develops a research agenda for quantitative reactive modeling and verification. Starting from the observation that discrete thinking has dominated computer science from its beginning and distinguishes it from many other engineering disciplines, Tom Henzinger aims in his ERC Advanced Grant "Quantitative Reactive Modeling" to replace classical Boolean correctness notions by quantitative fitness measures, which in particular are able to measure function, performance, and robustness of reactive and concurrent programs. Such quantitative measures would be much more suitable to express properties of real-life programs, as e.g. how much the behavior of programs differ from the desired one if the environment is faulty. The classical Boolean case will then be a special case in the entire quantitative setting. It is planned to not only apply the quantitative framework to multicore and cloud computing but also to systems biology as e.g. metabolic pathways in the cell.

Now it is your turn to explore this special issue and detect the new research approaches and results on rigorous software engineering. Enjoy!

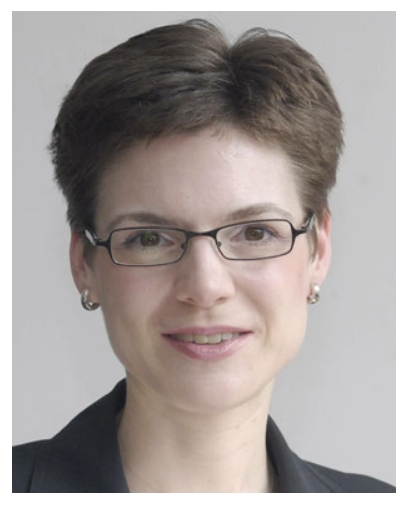

Sabine Glesner holds a Master of Science in Computer Science from the University of California, Berkeley, a diploma degree in Computer Science from the Technical University of Darmstadt, Germany, and a $\mathrm{Ph} . \mathrm{D}$. in Computer Science from the University of Karlsruhe, Germany. At the University of Karlsruhe, she also finished her habilitation, which qualified her as a university teacher. Currently, she is a full professor at the Technical University of Berlin, heading the chair Software Engineering for Embedded Systems. 